\title{
Avaliação da percepção de dor em recém-nascidos por profissionais de saúde de unidade neonatal
}

\section{Evaluation of pain perception in newborns by neonatal health professionals}

\author{
Ana Claudia Garcia Marques ${ }^{1 *}$ (D), Zeni Carvalho Lamy ${ }^{1}$ (D), \\ João Batista Santos Garcia² (D), Laura Lamas Martins Gonçalves ${ }^{1}$ (D), \\ Daniela Santos Bosaipo ${ }^{3}$ (D), Hanna Danielle Corrêa da Silva ${ }^{3}$ (D), \\ Taiana Mara Roma ${ }^{3}$ (D), Mariana Azevedo Sousa ${ }^{3}$ (D), Fernando Lamy Filho ${ }^{4}$
}

\begin{abstract}
Resumo
Introdução: A crescente utilização de recursos de terapia intensiva neonatal para manter a vida dos recém-nascidos enfermos pode gerar estímulos dolorosos, causando sofrimento a eles. Porém, nem sempre essa dor é percebida pelos profissionais envolvidos no cuidado, embora já exista um interesse crescente por parte deles nesse sentido. Objetivo: Avaliar a percepção de dor do recém-nascido por profissionais de saúde que atuam em unidade neonatal. Método: Pesquisa qualitativa do tipo exploratória, realizada em um hospital de referência em cuidado neonatal de uma capital do Nordeste. Foram realizadas entrevistas semiestruturadas com todos os profissionais que atuavam na Unidade de Terapia Intensiva Neonatal e/ou na Unidade de Cuidados Intermediários em cuidado direto com o recém-nascido. Utilizou-se da análise de conteúdo na modalidade temática, e os resultados foram agrupados em categorias. Resultados: Foram realizadas 36 entrevistas. Os profissionais entrevistados reconheceram que a dor neonatal foi historicamente negligenciada e que hoje as evidências científicas comprovam sua existência. Foram identificadas carência na formação no tema e fragilidade na aplicação do conhecimento na prática. Conclusão: Os profissionais têm conhecimento sobre a dor neonatal, entretanto quase não utilizam os parâmetros fisiológicos e possuem pouco conhecimento sobre a literatura científica atual. O principal desafio diz respeito à necessidade de uso sistemático de escalas que garantam a avaliação da dor.
\end{abstract}

Palavras-chave: dor; recém-nascido; percepção da dor; equipe de assistência ao paciente.

\begin{abstract}
Background: The increasing use of neonatal intensive care resources to maintain the lives of sick infants can generate painful stimuli and cause distress for them. However, this pain is not always perceived by the professionals involved in the care, although there is already an increasing interest on the part of the professionals involved in care. Objective: To evaluate the perception of health professionals, who works in a neonatal unit related to newborn pain. Method: Qualitative research of the exploratory type was carried out in a reference hospital in neonatal care of a capital of the Northeast. Semi-structured interviews were conducted with all professionals working in the Neonatal Intensive Care Unit and / or Intermediate Care Unit in direct care of the newborn. Content Analysis was used in the thematic modality and the results were grouped into categories. Results: 36 interviews were

`Departamento de Saúde Pública, Universidade Federal do Maranhão - São Luís (MA), Brasil.

²Departamento de Medicina II, Universidade Federal do Maranhão, São Luís (MA), Brasil.

3Universidade Federal do Maranhão - São Luís (MA), Brasil.

${ }^{4}$ Departamento de Medicina III, Universidade Federal do Maranhão - São Luís (MA), Brasil.

Trabalho realizado em um Hospital público - São Luís (MA), Brasil.

Endereço para correspondência: Ana Claudia Garcia Marques, Rua D, quadra L, Casa 02, Condomínio Lago Azul - Jardim Atlântico Turu - CEP: $65067-110$ - São Luís (MA), Brasil - E-mail: anaclaudia_marques@hotmail.com

Fonte de financiamento: Fundação de Amparo à pesquisa e Desenvolvimento científico do Maranhão (FAPEMA) e Coordenação de Aperfeiçoamento de Pessoal de Nível Superior (CAPES) - concessão da bolsa de mestrado, em momentos distintos.

Conflito de interesses: nada a declarar.
\end{abstract}


conducted. The professionals interviewed acknowledged that neonatal pain was historically neglected and that scientific evidence today confirms its existence. It was identified lack in the training in the theme and fragility in the application of the knowledge in the practice. Conclusion: The professionals have knowledge about neonatal pain; however they still use little physiological parameters and have little knowledge of current scientific literature. The main challenge is the need for systematic use of scales that guarantee the evaluation of pain.

Keywords: pain; newborn; perception of pain; patient care team.

\section{INTRODUÇÃO}

Até os anos 1980, a dor na criança, no lactente e no recém-nascido $(\mathrm{RN})$ não era diagnosticada de forma sistemática e, frequentemente, deixava de ser tratada ${ }^{1}$. Embora essa situação tenha evoluído, atualmente, com o avanço do conhecimento e o desenvolvimento tecnológico, também se observa o emprego crescente de procedimentos invasivos potencialmente dolorosos para manter a vida dos recém-nascidos gravemente enfermos.

A incapacidade dos recém-nascidos em verbalizar a dor é um desafio, fazendo com que dependam de cuidadores e ferramentas para avaliá-los e controlá-la ${ }^{2}$. Isso assume papel fundamental, uma vez que já é evidente na literatura a existência de associação entre as experiências de dor neonatal em recém-nascidos prematuros e o impacto negativo no crescimento pós-natal e no desenvolvimento cerebral, além de efeitos adversos em relação à atenção e ao desenvolvimento cognitivo, emocional e motor ${ }^{3-5}$. Em resposta a isso, o Ministério da Saúde (MS) recomendou o uso das escalas para avaliação com a validação de critérios objetivos da dor no recém-nascido (RN) para auxiliar nos processos de identificação e tratamento da dor. Segundo o protocolo do MS, a avaliação da dor deve ser repetida regularmente, de forma sistemática, independentemente da escala utilizada, devendo ser considerada como um quinto sinal vital ${ }^{6}$.

Ocorre que a avaliação da dor pode ser influenciada por uma série de fatores relacionados às características dos próprios observadores, como idade, gênero, experiência prévia pessoal com dor, entre outros. Além das características de quem observa a dor do RN, fatores inerentes ao paciente, como presença de dano tecidual e gravidade do diagnóstico, também podem alterar a inferência da presença de dor pelo observador. Sendo assim, a percepção sobre a dor pode ser caracterizada como uma experiência que alcança várias dimensões, pois é influenciada por fatores afetivos e motivacionais ${ }^{7,8}$. A subjetividade envolvida na avaliação do evento doloroso em recém-nascidos, caracterizada pela incapacidade do neonato em verbalizar, aliada à falta de treinamento profissional específico, dificulta o diagnóstico e o tratamento adequados da dor neonatal na prática diária9.

Portanto, torna-se necessário compreender a percepção desses profissionais sobre a dor em recém-nascidos para influenciar na construção de conhecimentos que possibilitem mudanças de práticas de saúde voltadas para o recém-nascido exposto a estímulos dolorosos. Este estudo teve como objetivo avaliar a percepção de profissionais de saúde sobre a dor no recém-nascido e suas perspectivas de enfrentamento do problema.

\section{MÉTODO}

Trata-se de uma pesquisa descritiva e analítica, com abordagem qualitativa do tipo exploratória, considerando a natureza do objeto a ser investigado.

A pesquisa foi realizada em um hospital universitário do Nordeste brasileiro, que oferece serviços de alta complexidade e é referência quanto à assistência para acadêmicos e profissionais. Os dados foram coletados no período de novembro de 2013 a novembro de 2014.

O Serviço de Neonatologia conta com 40 leitos distribuídos entre a Unidade de Terapia Intensiva Neonatal (UTIN), a Unidade de Cuidado Intermediário Neonatal Convencional (UCINCo) e a Unidade de Cuidado Intermediário Neonatal Canguru (UCINCa).

Os critérios de inclusão foram trabalhar há mais de um ano na Unidade Neonatal, possuir nível superior completo, realizar procedimentos e/ou ter contato direto com o recém-nascido.

A partir dos critérios de inclusão, foram identificados 38 profissionais. No entanto, no período de realização da coleta de dados, duas enfermeiras não aceitaram participar da entrevista. Dessa forma, a coleta de dados foi realizada com 36 profissionais, sendo 13 médicos, 15 enfermeiros, 5 fisioterapeutas, 2 fonoaudiólogos e 1 terapeuta ocupacional. A idade dos participantes variou de 25 a 60 anos, e a maioria era do sexo feminino, casada ou vivendo em união estável e tinha, no mínimo, um filho.

A técnica de coleta de dados utilizada foi entrevista semiestruturada, realizada a partir de dois instrumentos: um questionário estruturado, aplicado individualmente, antes da entrevista, visando conhecer aspectos sociodemográficos; e um roteiro de entrevista semiestruturada, abordando temas relativos ao trabalho com os recém-nascidos na Unidade Neonatal, ao conhecimento relacionado à dor e às escalas de avaliação no período neonatal bem como à atuação diante da dor do recém-nascido.

As entrevistas foram realizadas por duas entrevistadoras, sendo uma delas a pesquisadora responsável pela pesquisa. As transcrições foram realizadas por alunos de graduação, bolsistas de iniciação científica, sendo posteriormente revisadas exaustivamente pela responsável por esta pesquisa. As falas foram 
transcritas de forma literal e assim analisadas. Os nomes dos entrevistados foram substituídos por nomes fictícios.

A análise das entrevistas deu-se a partir da análise de conteúdo que, segundo Bardin ${ }^{10}$, é um conjunto de técnicas que visa obter, por procedimentos sistemáticos, as condições de produção e recepção das mensagens. Entre as quatro modalidades da análise de conteúdo foi utilizada a modalidade temática, na qual o pesquisador agrupa os dados do campo por temas, a partir dos seguintes passos: 1 . Pré-análise - transcrição transformando as falas em texto, leitura flutuante, exaustiva e interrogativa do material, apreensão das ideias centrais e determinação das unidades de registro e de contexto; 2 . Fase de categorização e exploração do material - apreensão dos núcleos de compreensão do texto buscando expressões ou palavras significativas, em torno das quais as falas se organizam; 3 . Interpretação - inferências e interpretações relacionando os núcleos de sentido com literatura vigente relativa à questão estudada ${ }^{10}$.

Esta pesquisa foi aprovada pelo Comitê de Ética em Pesquisa sob o protocolo No 446.969 , de $01 / 11 / 2013$. As entrevistas foram realizadas após assinatura do Termo de Consentimento Livre e Esclarecido (TCLE), e a identidade dos entrevistados foi preservada para assegurar seu anonimato.

\section{RESULTADOS E DISCUSSÃO}

Em relação à formação profissional, a maioria tinha mais de 5 anos de formação. Apenas 1 profissional da área de Enfermagem com 10 anos de formação não tinha nenhuma pós-graduação, enquanto 14 profissionais (enfermeiras, fisioterapeutas e fonoaudióloga) tinham especialização lato sensu, 12 cursaram residência médica e multiprofissional, 7 eram mestres e 2 eram doutores.

Os profissionais das diferentes categorias referiram que percebiam a dor no recém-nascido principalmente a partir de manifestações comportamentais, como expressão facial e choro, mas também mencionaram as manifestações fisiológicas, sendo as mais citadas: frequência cardíaca, saturação de oxigênio e respiração. Nos exemplos a seguir, um médico e uma enfermeira falam a respeito:

A face dele. Eu gosto muito de olhar. Também quando eu vou botar o estetoscópio na criança, eu gosto de ficar observando a frequência cardíaca antes e depois. E a saturação às vezes também. Mas, eu geralmente observo mais pela face (Médica Orquídea).

Movimentos [...] Ele tá com movimento irregular, chorando, fazendo careta, e às vezes ele dessatura quando tá sentindo dor (Enfermeira Rosa).

Outros estudos também demonstraram que, para os profissionais de saúde, a presença da dor no $\mathrm{RN}$ foi identificada por meio de alterações nos parâmetros fisiológicos e comportamentais, e os mais citados foram o choro e a expressão facial ${ }^{11-14}$. Respostas fisiológicas também são frequentemente utilizadas (frequência cardíaca, saturação e frequência respiratória $)^{11,15}$. Oliveira et al. observaram em seu estudo que a equipe também avaliava o humor do recém-nascido, além dos parâmetros comportamentais já descritos ${ }^{16}$. As alterações cardiovasculares e respiratórias foram as modificações fisiológicas mais citadas pelos profissionais, achados muito próximos aos encontrados nesses estudos.

A soberania da avaliação comportamental diante da avaliação de manifestações fisiológicas constatada na fala dos diferentes profissionais sugere pouco conhecimento da literatura científica atual, tendo em vista que esta aponta que os parâmetros fisiológicos são tão importantes quanto os comportamentais na avaliação da dor. Muitas vezes, o recém-nascido muito doente pode não expressar mudanças comportamentais.

Os pequenos sinais, como hipoatividade e alteração no estado de sono/vigília, para os recém-nascidos doentes são importantes indicadores de dor neonatal e foram citados por uma médica e por uma enfermeira entrevistadas:

Aqueles que estão muito estressados, ou que estão muito largados, é porque estão sentindo dor [...]. Vejo logo o procedimento que vai ser feito ou que está sendo feito (Médica Bromélia).

Ele pode, de repente ter uma diminuição na saturação de oxigênio, alguns parâmetros vão diminuir. Ou às vezes ele tá tão cansado de tudo e apaga, dorme demais depois de tanta dor, de tanto sofrimento, e apaga (Enfermeira Jasmin).

Para a maioria dos profissionais entrevistados, o choro significa dor, porém muitos recém-nascidos não a manifestam assim, a exemplo daqueles entubados. Outra questão é que nem sempre o choro está relacionado à dor. Essa questão foi relatada por alguns entrevistados conforme no exemplo a seguir:

O choro é um momento que te chama atenção para a dor. Mas nem sempre o choro representa que o bebê está com dor. Porque a gente sabe que o choro às vezes é uma fralda que está molhada, cheia de fezes [...] (Fisioterapeuta Girassol).

O choro para o RN é uma das maneiras de comunicação rotineira e se manifesta quando há algum desconforto, podendo ou não ser uma reação dolorosa ${ }^{17}$. No entanto, em um estudo realizado com profissionais de Enfermagem, o choro foi o principal responsável pelos registros de dor nos bebês, mesmo sendo um parâmetro pouco específico para avaliação da dor, quando usado isoladamente ${ }^{18}$.

Foi apontada pelos profissionais uma diferença da percepção de dor entre as diferentes categorias. Muitos entrevistados mencionaram que a equipe de Enfermagem é a mais atenta à dor no recém-nascido.

Como a enfermagem trabalha diretamente com esse $R N$, ela manuseia mais, cuida mais, então elas acabam observando melhor aquela criança do que outra pessoa, como o médico, o fisioterapeuta [...] (Fisioterapeuta Azaleia).

Quem está mais junto, até para perceber a dor, é a equipe de enfermagem [...] (Médica Gerbera). 
Para Costa et al., o manejo clínico no alívio da dor neonatal deve ser uma constante preocupação dos profissionais de saúde, especialmente da equipe de Enfermagem que é responsável pelo cuidado direto do RN. Esses autores destacam que o enfermeiro que deve estar apto a detectar qualquer alteração ou manifestação de dor e aliviá-la da melhor forma possível ${ }^{2}$.

Foi ainda frequentemente referido que a dor neonatal não era valorizada por médicos especialistas que respondem pareceres e realizam procedimentos na Unidade Neonatal, com destaque para os cirurgiões.

Esses especialistas, não tem uma visão da dor, como a gente tem, apesar de trabalharem com recém-nascidos também, eles dão menos importância à dor do que os neonatologistas (Médica Margarida).

Os cirurgiões precisam ser sensibilizados, porque dos profissionais, eu acho que esses são os que menos estão atentos para essa questão da dor. Então às vezes a gente percebe, assim, que eles não... Não são todos, lógico. Mas às vezes eles chegam e querem fazer tudo rápido, não esperam nem [...] (Enfermeira Tulipa).

Para Melo et al., profissionais que não atuam especificamente em Neonatologia têm uma percepção diminuída ou incipiente em relação à dor do recém-nascido ${ }^{19}$. Reconhecer a dor neonatal requer habilidade e sensibilidade, especialmente por se tratar de uma população que se comunica de forma não verbal. Maia e Coutinho também afirmam que profissionais que não trabalham diretamente em Unidade de Terapia Intensiva Neonatal não reconhecem a dor como sinal vital ${ }^{20}$. Segundo os autores, a experiência de trabalho na UTIN tem sido percebida como importante fator inicial de sensibilização à dor do neonato, embora ainda sejam necessários avanços, como apontado neste trabalho.

Alguns entrevistados mencionaram o conhecimento adquirido em cursos como importante para melhorar a identificação da dor neonatal e, consequente, ampliar a percepção de profissionais.

A educação continuada, apesar de todas as falhas, ajudou a gente a identificar quando o bebê estava com dor (Enfermeira Violeta).

Estudos constataram mudanças na percepção e no tratamento da dor após a implementar intervenção educativa em profissionais de uma Unidade Neonatal ${ }^{21-23}$. Segundo Almeida, cada geração possui, aparentemente, características distintas relacionadas ao modo como trabalham e adquirem conhecimento, assim como é imprescindível complementar o ensino tradicional com outras estratégias. Nesse contexto, o uso de vídeos instrucionais objetivos, com base em evidências e disponíveis no meio on-line, poderia favorecer a interação, o engajamento e a promoção do aprendizado cognitivo e afetivo, além de guiar a tomada de decisão desses profissionais e melhorar a qualidade do cuidado ${ }^{24}$.

Contudo, a maioria dos profissionais referiu ainda que há lacunas no conhecimento dessa temática durante a graduação e até mesmo em especializações.

Nunca tive contato com esse assunto na graduação, não [...]. Eu fui ter contato depois. Na prática mesmo. É na residência, que a gente tem contato com a UTI mesmo, que a gente foi falar sobre o assunto, que eu fui ter noção disso (Médica Amarílis).

Essa constatação pode contribuir para a compreensão da dificuldade de mudanças de práticas já identificadas.

$\mathrm{O}$ acesso ao conhecimento científico atualizado por parte da equipe e a sistemática colocação em análise dos processos de trabalho podem contribuir para uma ressignificação dos saberes e das práticas ${ }^{25}$. A dor tem relação direta com variáveis culturais, sociais, ambientais, psicológicas e educacionais, e todas necessitam ser pelo menos discutidas quando se propõe a elaboração de protocolos e rotinas para o manejo da dor no ambiente hospitalar e, principalmente, na Unidade Neonatal ${ }^{26}$.

Acredita-se também que a inserção dessa temática nos currículos de graduação e pós-graduação e nas atividades de educação permanente, assim como a adoção de protocolos para toda a equipe da Unidade Neonatal e o planejamento de ações para o manejo da dor, consista em importantes estratégias para o aprimoramento do processo de trabalho com vistas à prevenção e ao tratamento da dor neonatal.

Os resultados obtidos nesta pesquisa demonstram que, apesar da importância da percepção individual dos profissionais quanto a sinais indicativos de dor no recém-nascido e de eles afirmarem ter conhecimento sobre a dor neonatal, poucos utilizam os parâmetros fisiológicos como indicativos de dor, além de possuírem pouco conhecimento da literatura científica atual ${ }^{27,28}$.

Diante dessa realidade e levando em conta a variabilidade individual na identificação da dor no recém-nascido, o principal desafio diz respeito à necessidade da implementação de protocolos diários que incorporem métodos e instrumentos validados (escalas de dor) que permitam aos profissionais da instituição avaliar a dor do recém-nascido criticamente doente de maneira sistemática, a fim de que, uma vez identificada, ela possa ser tratada da melhor forma possível.

\section{REFERÊNCIAS}

1. Brasil. Ministério da Saúde. Secretaria de Atenção à Saúde. Departamento de Ações Programáticas Estratégicas. Atenção humanizada ao recém-nascido de baixo peso: Método Canguru. 3. ed. Brasília, DF; 2017.

2. Costa KF, Alves VH, Dames LJP, Rodrigues DP, Barbosa MTSR, Souza RRB. Manejo clínico da dor no recém-nascido: percepção de enfermeiros da unidade de terapia intensiva neonatal. J Res: Fundam Care. 2016;8(1):3758-3769.
3. Costa T, Rossato LM, Bueno M, Secco IL, Sposito NPB, Harrison D, et al. Nurses' knowledge and practices regarding pain management in newborns. Rev Esc Enferm USP. 2017;51:1-7. http://dx.doi.org/10.1590/s1980220x2016034403210. PMid:28403366.

4. Victoria NC, Murphy AZ. Exposure to early life pain: Long term consequences and contributing mechanisms. Curr Opin Behav Sci. 2016;7:61-8. http:// dx.doi.org/10.1016/j.cobeha.2015.11.015. PMid:27525299. 
5. Walker SM. Translational studies identify long-term impact of prior neonatal pain experience. Pain. 2017;158(1 Suppl):S29-4. http://dx.doi. org/10.1097/j.pain.0000000000000784. PMid:28106669.

6. Brasil. Ministério da Saúde. Secretaria de Atenção à saúde. Departamento de Ações Programáticas e Estratégicas. Atenção à saúde do recém-nascido: guia para os profissionais de saúde (Vol. 4). Brasília, DF; 2011.

7. Bottega FH, Benetti ERR, Benetti PE, Benetti PE, Gomes JS, Stumm EMF Avaliação da dor em neonatos e crianças em terapia intensiva. J Res: Fundam Care. 2014;6(3):909-917.

8. Christoffel MM, Castral TC, Daré MF, Montanholi LL, Gomes ALM, Scochi CGS. Atitudes dos profissionais de saúde na avaliação e tratamento da dor neonatal. Esc Anna Nery. 2017;21(1):1-8.

9. Scherman E, Johansson L, Gradin M, Johannes VDB, Eriksson M. Procedural pain in neonates: do nurses follow national guidelines? A survey to Swedish neonatal units. J Neonatal Nursing. 2014;20(1):31-6.

10. Bardin L. Análise de conteúdo. São Paulo: Edições 70; 2011.

11. Harrison D, Bueno M, Reszel J. Prevention and management of pain and stress in the neonate. Res Rep Neonatol. 2015;5:9-1.

12. Pinheiro IO, Lima FET, Magalhães FJ, Farias LM, Sherlock MSM. Avaliação da dor do recém-nascido através da escala Codificação da Atividade Facial Neonatal durante o exame de gasometria arterial. Rev Dor. 2015;16(3):176-80.

13. Andreazza MG, Motter AA, Cat ML, Silva RPGVC. Percepção da dor em neonatos pela equipe de enfermagem de unidade de terapia intensiva neonatal. Rev Bras Pesq. 2017; 19(4):133-139.

14. Araujo GC, Miranda JOF, Santos DV, Camargo CL, Sobrinho CLN, Rosa DOS. Dor em recém-nascidos: identificação, avaliação e intervenções. Rev Baiana Enferm. 2015;29(3):261-70. http://dx.doi.org/10.18471/rbe. v29i3.13695.

15. Lotto CR, Linhares MBM. Contato "Pele a Pele" na prevenção de dor em bebês prematuros: revisão sistemática da literatura. Trends Psychol. 2018;26(4):1699-1713

16. Oliveira RM, Silva AVS, Chaves EMC, Salles NC. Avaliação comportamental e fisiológica da dor em recém-nascidos pelos profissionais de enfermagem. Rev Min Enferm. 2010;14(1):19-24.

17. Rodrigues JB, Souza DSB, Werneck AL. Identificação e avaliação da percepção dos profissionais de enfermagem em relação a dor/desconforto do recém-nascido. Arq Ciênc Saúde. 2016;23(1):27-31.
18. Elias LSDT, Cajigas C, Thimóteo BS, Barbisan GG, Cavaleti JB, Alves TM. Avaliação da dor na unidade neonatal sob a perspectiva da equipe de Enfermagem em um hospital no noroeste paulista. Rev Cuidarte Enfermagem. 2016; 10(2):156-161.

19. Melo GM, Rebouças CBA, Cardoso MVLML, Farias LM. Nursing team communication with regard pain in newborns: a descriptive study. Online Braz j Nurs. 2013;12(2):462-70.

20. Maia ACA, Coutinho SB. Fatores que influenciam a prática do profissional de saúde no manejo da dor do recém-nascido. Rev Paul Pediatr. 2011;29(2):270-6.

21. Aymar L, Lima LS, Santos CM, Moreno EA, Coutinho SB. Pain assessment and management in the NICU: analysis of on educational intervention for health professionals. J Pediatr. 2014;90(3):308-15. http://dx.doi.org/10.1016/j. jped.2013.09.008. PMid:24560962.

22. Christoffel MM, Castral TC, Daré MF, Montanholi LL, Scochi CG. Conhecimento dos profissionais de saúde na avaliação e tratamento da dor neonatal. Rev Bras Enferm. 2016;69(3):552-8. http://dx.doi.org/10.1590/00347167.2016690319i. PMid:27355306.

23. Silva LM, Silva AS, Andrade EWOF, Azevedo AP, Costa FWS, Azevedo MKP. A avaliação da dor do recém-nascido realizada pela equipe de enfermagem. Rev UNINGÁ Review. 2018;33(1):1-11.

24. Almeida HCC, Candido LK, Harrison D, Bueno M. Seja Doce com os Bebês: avaliação de vídeo instrucional sobre manejo da dor neonatal por enfermeiros. Rev Esc Enferm USP. 2018;52:1-7. http://dx.doi.org/10.1590/ s1980-220x2017033903313. PMid:29947704.

25. Silva LM, Silva AS, Andrade EWOF, Azevedo AP, Costa FWS, Azevedo MKP. A avaliação da dor do recém-nascido realizada pela equipe de enfermagem. Rev UNINGÁ Review. 2018;33(1): 1-11.

26. Martins, SW; Enumo, SRF; Paula, KMP. Manejo da dor neonatal: influência de fatores psicológicos e organizacionais. Estud Psicol. 2016;33(4):633-644.

27. Committee on Fetus and Newborn \& Section on Anesthesiology and Pain Medicine. Prevention and management of procedural pain in the neonate: An update. Pediatrics. 2016;137(2):1-15.

28. Oliveira IM, Castral TC, Cavalcante MMFP, Carvalho JC, Daré MF, Salge AKM. Conhecimento e atitude dos profissionais de enfermagem sobre avaliação e tratamento da dor neonatal. Rev Eletr Enf. 2016;18;1-11. http:// dx.doi.org/10.5216/ree.v18.36782.

Recebido em: Apr. 12, 2018 Aceito em: Apr. 05, 2019 